\title{
Editorial
}

Z Gerontol Geriat 2013 · 46:388-389

DOI 10.1007/s00391-013-0515-7

Online publiziert: 8. Juni 2013

(c) Springer-Verlag Berlin Heidelberg 2013
K. Pils

SMZ-Sophienspital, Wien

\section{Ernährung im Alter}

erkrankungen zu. Auch übergewichtige Menschen können mangelernährt sein. Meist fehlt jedoch das Bewusstsein für dieses scheinbare Paradoxon. So kann die Abnahme der Muskelmasse durch Mangel an essenziellen Aminosäuren und adäquatem Training zu Funktionsverlust und Pflegeabhängigkeit führen [1].

Dieses Themenheft versucht, sich dem breiten Feld der Ernährungsstörungen im Alter und deren Folgen zu widmen. Unabhängig davon, ob es sich um Mangelernährung oder Adipositas handelt, Ernährung ist nur ein Teil der Faktoren, die zu einem weitgehend autonomen Alter(n) beitragen. So führt vor allem der Eiweißmangel verbunden mit reduzierter körperlicher Aktivität zu Sarkopenie und letztlich zum Einstieg in die Inkompetenzkaskade bis hin zu Frailty und steigendem Bedarf an Pflegeabhängigkeit. Vitamin D ist in seiner aktiven Form an zahlreichen Stoffwechselprozessen beteiligt. Durch den Mangel an Aufnahme mit der Nahrung, an Resorption einerseits und verminderter Metabolisierung andererseits, zeigen fast alle älteren Menschen eine ausgeprägte Defizienz. Diese nimmt mit der Aufnahme in eine betreuende Einrichtung zu. Reaktiver Rückzug und körperliche Inaktivität begünstigen die Entwicklung von Osteoporose, Sturzrisiko und Frakturen.

Mit den vorliegenden Artikeln soll die Komplexität angeschnitten und zur weiterführenden Diskussion angeregt werden. Die Deutsche Gesellschaft für Ernährungsmedizin veröffentlicht beginnend mit April eine Artikelserie der neuen Leitlinien. Diese fassen strukturiert das aktuelle Wissen zusammen. Dennoch müssen auch politische und gesundheitspolitische Maßnahmen ergriffen werden, wenn das Bewusstsein der Ärzte, der Pflegepersonen und vor allem der Bevölkerung verändert werden soll.

Auch wenn die Politik sich der Problematik der Fehlernährung durchaus be-
Diese scheinbar banalen Gedanken haben einen wesentlichen Einfluss auf zwei parallel bestehende Phänomene der letzten Jahrzehnte: die Mangelernährung selbst gewählt oder konsekutiv - und die Obesitas. Diese Polarität verändert das Körperbewusstsein von Generationen. Die Konsequenzen können wir aus heutiger Sicht noch nicht abschätzen.

Im Alter gewinnt die Mangelernährung an Bedeutung. Dabei ist allerdings nicht das seit Jahren konstante Körpergewicht von Bedeutung, sondern vielmehr der unbeabsichtigte signifikante Gewichtsverlust. Dieser kann durch eine Verschlechterung des Gesundheitszustands als Folge von Krankenhausaufenthalten, Multimorbidität, Multimedikation oder der sozialen Isolation und/oder reaktiven Depression entstehen.

Gleichzeitig nimmt die Zahl der Übergewichtigen mit zivilisatorischen Folge-

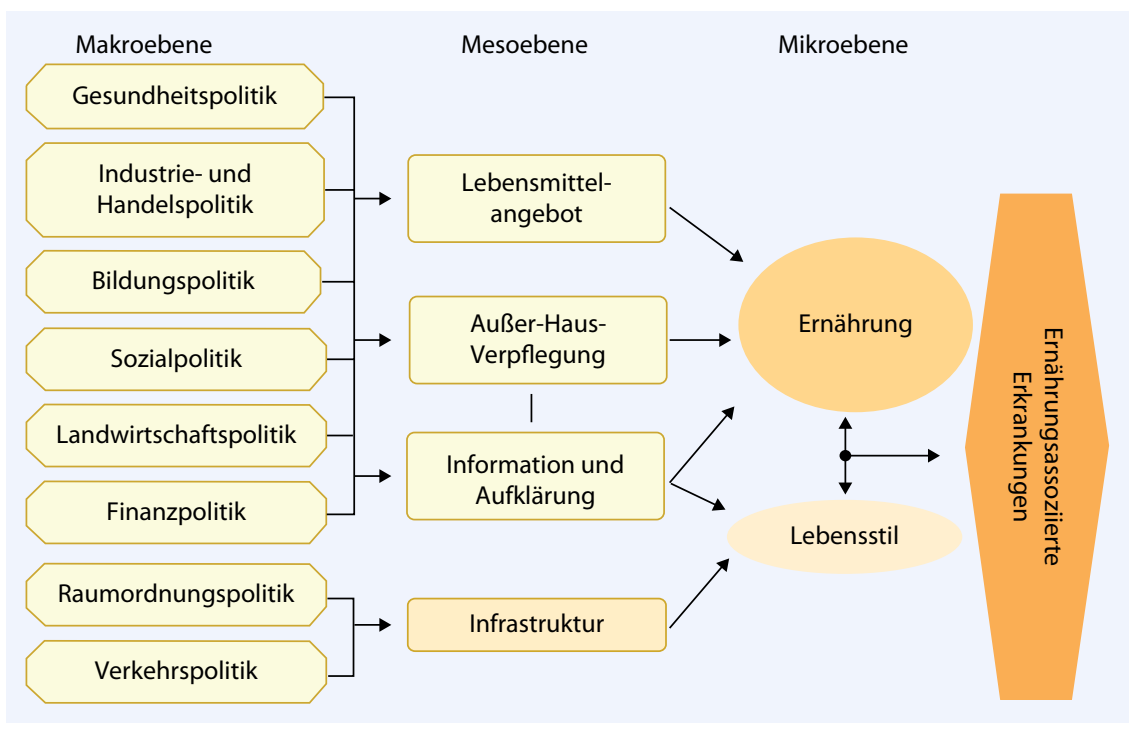

Abb. $1 \Delta$ Nationaler Aktionsplan Ernährung 2012. (Aus [2], mit freundl. Genehmigung des Bundesministeriums für Gesundheit, Österreich) 
wusst ist, werden die Empfehlungen noch nicht nachhaltig in die Praxis umgesetzt. Der Nationale Aktionsplan Ernährung Österreich [2] spannt den längst notwendigen breiten Bogen von der Kindheit bis ins hohe Alter und fokussiert auf die Komplexität von Lebensstilfragen (• Abb. 1).

Veränderungen dürfen nicht nur auf die Mikroebene - die Personen und ihre Familien selbst - und die Mesoebene Krankenhaus, Pflegeheimbetreiber und ambulante Pflegedienste - fokussieren. Langfristig müssen die Politik und die ihr anvertraute Makroebene ein Umdenken einleiten. Es müssen realistische und sektorenübergreifende Konzepte für eine gesündere Umwelt und Stärkung des individuellen Gesundheitsbewusstseins, soweit verfügbar evidenzbasiert, etabliert werden.

Langfristig sollten sich Interventionsmodelle wie von Stingel et al. [3] an mehreren Outcome-Varianten orientieren: Es geht nicht nur um biomedizinische Parameter unter Berücksichtigung des individuellen Willens, sondern auch um sozioökonomische Modelle. Als Basis für weitere Begleitforschung wurde zunächst eine gemeinsame Sprache geschaffen [4].

Darauf aufbauend wurden Ernährungsempfehlungen für Menschen in unterschiedlichen Lebensphasen und Bedürfnisgraden entwickelt.

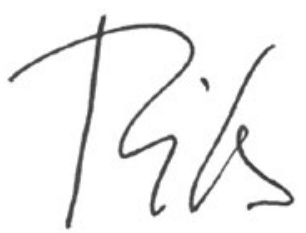

Katharina Pils

\section{Korrespondenzadresse}

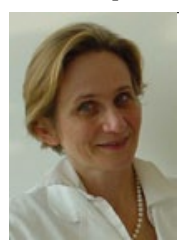

Prim. Dr. K. Pils

SMZ-Sophienspital

Apollogasse 19, 1070 Wien

Österreich

Katharina.pils@wienkav.at

\section{Literatur}

1. Beasley JM, LaCroix AZ, Neuhouser ML et al (2010) Protein intake and incident frailty in the Women's Health Initiative Observational Study. JAGS 58:1063-1071

2. Bundesministerium für Gesundheit (2012) Nationaler Aktionsplan Ernährung 2012. BMG Österreich. http://bmg.gv.at/cms/home/attachments/1/3/0/CH1046/CMS1264514565545/nape_2012_final.pdf

3. Stingel K, Schütz T, Koller M et al (2013) Leitlinie der Deutschen Gesellschaft für Ernährungsmedizin (DGEM). Methodik zum Leitlinien-Update Klinische Ernährung. Aktuel Ernahrungsmed 38:90-96

4. Valentini L, Volkert D, Schütz T et al (2013) Leitlinie der Deutschen Gesellschaft für Ernährungsmedizin (DGEM). DGEM-Terminologie in der Klinischen Ernährung. Aktuel Ernahrungsmed 2013; 38:97111 